\title{
Co-existence of Congenital Adrenal Hyperplasia and Bartter syndrome due to maternal uniparental isodisomy of HSD3B2 and CLCNKB mutations
}

Dinesh Giri', 2, Detlef Bockenhauer ${ }^{3}$, Charu Deshpande ${ }^{4}$, John C Achermann ${ }^{5}$, Norman F Taylor ${ }^{6}$, Gill Rumsby ${ }^{7}$, Henry Morgan ${ }^{8}$, "Senthil Senniappan ${ }^{8},{ }^{*}$ Michal Ajzensztejn ${ }^{4}$

*joint last authors

1'Bristol Royal Hospital for Children, Bristol, United Kingdom

${ }^{2}$ Department of Translational Health Sciences, University of Bristol

${ }^{3}$ Great Ormond Street Hospital for Children, London, United Kingdom

${ }^{4}$ Guy's and St Thomas' Hospital NHS Foundation Trust, London, United Kingdom

${ }^{5}$ UCL GOS Institute of Child Health, University College of London, London, United Kingdom

${ }^{6}$ King's College Hospital, London, United Kingdom

${ }^{7}$ University College of London Hospital NHS Foundation Trust, London, United Kingdom

${ }^{8}$ Alder Hey Children's Hospital, Liverpool, United Kingdom

\section{Corresponding author:}

Dr Dinesh Giri

Consultant Paediatric Endocrinologist \& Honorary Senior Lecturer

Bristol Royal Hospital for Children \& University of Bristol

Education centre

Upper Maudlin Street

Bristol

BS2 8BJ

Email: Dinesh.Giri@UHBristol.nhs.uk

Phone: +441173429336

Key words: HSD3B2 deficiency, Bartter syndrome, maternal isodisomy

Running title: Co-existence of dual opposing pathologies-CAH and Bartter syndrome

Full article

https://www.karger.com/Article/Abstract/507577

PMID: 32506065 DOI: 10.1159/000507577 


\section{Established facts}

- HSD3B2 enzyme deficiency is one of the rarest forms of congenital adrenal hyperplasia associated with salt-wasting due to hypoaldosteronism, hypoglycaemia due to glucocorticoid deficiency and undervirilisation of the external genitalia in genotypic males due to androgen deficiency.

- Bartter syndrome causes hypokalemic, hypochloraemic metabolic alkalosis by activation of the renin-angiotensin system.

\section{Novel insights}

- The co-existence of HSD3B2 enzyme deficiency and Bartter syndrome can result in a state of persistent hypokalemic alkalosis suggestive of high mineralocorticoid activity.

- Identification of a rare homozygous mutation in an offspring of nonconsanguineous parents should raise suspicion of uniparental isodisomy. 


\section{Abstract}

\section{Introduction:}

We present a patient with co-existence of $3 \beta$-Hydroxysteroid dehydrogenase type 2 (HSD3B2) deficiency and Bartter Syndrome, a unique dual combination of opposing pathologies that has not been reported previously in the literature.

\section{Case:}

A female infant $(46, \mathrm{XX})$ born at $34 / 40$ weeks' gestation, weighing $2.67 \mathrm{Kg}(-1.54$ SDS) to non-consanguineous parents presented on day four of life with significant weight loss. Subsequent investigations revealed hyponatraemia, hypochloraemia, metabolic alkalosis, elevated 17-hydroxyprogesterone, ACTH and renin. Urine steroid profile suggested HSD3B2 deficiency, which was confirmed by the identification of a homozygous $H S D 3 B 2$ mutation. Due to the persistence of the hypochloraemic and hypokalemic alkalosis an underlying renal tubulopathy was suspected. Sequence analysis of a targeted tubulopathy gene panel revealed a homozygous deletion in $C L C N K B$, consistent with Bartter syndrome type 3. The mother was found to be heterozygous for both mutations in HSD3B2 and CLCNKB and the father negative for both. Single nucleotide polymorphism microarray analysis confirmed 2 segments of homozygosity on chromosome 1 of maternal ancestry, encompassing both HSD3B2 and CLCKNB.

\section{Discussion:}

Identification of a homozygous rare mutation in an offspring of non-consanguineous parents should raise suspicion of uniparental disomy, especially if the phenotype is unusual, potentially encompassing more than one disorder. The persistence of hypokalemic alkalosis, the biochemical fingerprint of hyperaldosteronism in a child with a form of $\mathrm{CAH}$ in which aldosterone production is severely impaired challenges 
our current understanding of mineralocorticoid-mediated effects in the collecting duct.

\section{Introduction}

Congenital Adrenal Hyperplasia $(\mathrm{CAH})$ comprises a group of disorders that are inherited in an autosomal recessive fashion due to deficiencies of enzymes required for cortisol biosynthesis [1]. The majority of $\mathrm{CAH}$ (about 90-95\%) are due to the deficiency of the 21-hydroxylase enzyme, while HSD3B2 enzyme deficiency is rare. HSD3B2 catalyses the conversion of pregnenolone to progesterone, a precursor of aldosterone and the conversion of 17-hydroxypregnenolone to 17hydroxyprogesterone, a precursor of cortisol [1]. In addition, it also catalyses the conversion of dehydroepiandrosterone (DHEA) to androstenedione, a precursor of testosterone [1]. HSD3B2 is encoded by $H S D 3 B 2$, with recessive loss-of-function mutations resulting in various degrees of salt-wasting in both sexes due to hypoaldosteronism and undervirilisation of the external genitalia in genotypic males due to reduced testosterone biosynthesis [2]. In genotypic females, it can cause mild virilisation due to the accumulation of the androgen DHEA, some of which gives rise to testosterone via conversion to androstenedione by the peripheral HSD3B1 isoform.

Bartter syndrome comprises a group of autosomal recessively inherited disorders, caused by defects in salt transport in the thick ascending loop of Henle (TAL) resulting in marked renal salt wasting. Mutations in six different genes (SLC12A1, KCNJI, CLCNKB, CLCNKA, BSND and MAGED2) have been implicated in different types of Bartter syndrome, all of which are characterised by severely reduced or absent salt transport across the TAL resulting in hypokalaemic metabolic alkalosis 
and elevated renin and aldosterone, although there may be some variabilities in the phenotype [3].

We present for the first time the co-existence of a unique combination of dual opposing pathologies contributed by the hypoaldosteronism in HSD3B2 enzyme deficiency and hyperreninism in Bartter Syndrome, due to maternal uniparental isodisomy (UPiD) of HSD3B2 and CLCNKB mutations.

\section{Case}

The patient is a baby girl, born at 34 weeks' gestation following spontaneous labour with a birth weight of $2.67 \mathrm{~kg}(-1.54 \mathrm{SDS})$ to a non-consanguineous ethnic white British mother and a British-Afro-Caribbean father. The pregnancy was uncomplicated and the antenatal scans were normal. She presented at 4 days of age with weight loss (12\%), jaundice and poor feeding. Her investigations revealed hyponatraemia $(127 \mathrm{mmol} / \mathrm{L})$, hypochloraemia $(87 \mathrm{mmol} / \mathrm{L})$, hyperkalaemia $(6.8$ $\mathrm{mmol} / \mathrm{L})$ and metabolic alkalosis $\left(\mathrm{pH} 7.54, \mathrm{PCO}_{2} 4.7 \mathrm{kpa}, \mathrm{PO}_{2} 5.2 \mathrm{kpa}\right.$, bicarbonate $29.9 \mathrm{mmol} / \mathrm{l})$. Further investigations showed an elevated renin of $2206 \mathrm{mU} / \mathrm{L}$ (5.4-30), an inappropriately low aldosterone of $169 \mathrm{pmol} / \mathrm{l}(1000-4500 \mathrm{pmol} / \mathrm{L})$ a high $\mathrm{ACTH}$ of $553 \mathrm{ng} / \mathrm{L}$. These suggested adrenal insufficiency in both mineralocorticoid and glucocorticoid pathways. 17-Hydroxyprogesterone was elevated at $>110 \mathrm{nmol} / \mathrm{L}$. She had female-typical external genitalia with no ambiguity and a normal female karyotype, 46,XX. Her blood pressure was normal. Other results included DHEAS: 7umol/L (0.9-11.6); the testosterone sample was insufficient. A urine steroid profile on a sample collected on day 12 (Table 1) showed a marked increase in 3ß-hydroxy3-ene steroids relative to cortisol metabolites, consistent with a diagnosis of HSD3B2 enzyme deficiency. She was commenced on hydrocortisone (10mg/m2/day), 
fludrocortisone and sodium chloride supplements. Samples collected at 14 months while receiving maintenance hydrocortisone in conjunction with a synacthen test showed the expected predominance of pregnenetriol, the major metabolite of 17hydroxypregnenolone. Table 1 compares values before and after synacthen and with published values to illustrate the differentiation from 21-hydroxylase deficiency, further discussed below. This was subsequently confirmed by sequencing of HSD3B2, which revealed an apparently homozygous $H S D 3 B 2$ mutation [c.745C $>\mathrm{T}$, p. Arg249*]. This mutation is not listed in ClinVar but the heterozygote allele is listed in gnomAD. As this a nonsense mutation, this will be expected to result in saltwasting as there will be no residual enzyme activity. Despite increasing the dose of fludrocortisone (maximum dose of $225 \mathrm{mcg} / \mathrm{m}^{2} /$ day) the plasma renin concentration persistently remained more than $500 \mathrm{mU} / \mathrm{L}$ (5.4-30). However, she started to develop persistent hypokalaemia as well as ongoing hypochloraemia and metabolic alkalosis. Due to the persistence of the hypokalaemic alkalosis, the dose of fludrocortisone was gradually weaned, but despite this she remained hypokalaemic with her plasma potassium concentrations consistently below $3 \mathrm{mmol} / \mathrm{L}$ and plasma renin concentration increasing to a maximum of $15,795 \mathrm{mU} / \mathrm{L}$. Potassium supplements were commenced $(2.5 \mathrm{mmol} / \mathrm{kg} /$ day) without much improvement of the plasma potassium concentration. A blood and urine electrolyte profile after withholding her fludrocortisone for 36 hours and sodium and potassium supplements for 24 hours with close monitoring of blood pressure and volume status showed persistence and worsening of hypokalaemia $(2.5 \mathrm{mmol} / \mathrm{L})$. The fractional excretion $(\mathrm{FE})$ of $\mathrm{Na}$ was $0.9 \%$, $\mathrm{FE}$ of $\mathrm{K} 19 \%$, $\mathrm{FE}$ of $\mathrm{Cl} 0.8 \%$, and the transtubular potassium gradient (TTKG) was 9.1, suggestive of renal potassium wasting in the collecting duct, usually mediated by aldosterone. Due to the unusual electrolyte constellation with 
hypokalaemic, hypochloraemic metabolic alkalosis in the background of an underlying mineralocorticoid deficiency, an underlying tubulopathy was suspected. Screening of 37 genes associated with tubulopathy (Tubulopathy Next Generation Sequencing panel) identified a homozygous deletion from exon 1 to 19 of $C L C N K B$ consistent with a diagnosis of Bartter syndrome type 3 [4].

Parental genetic testing showed that the mother was heterozygous for both the mutations in $H S D 3 B 2$ and $C L C N K B$ and the father did not carry any mutation in these 2 genes. The co-existence of two rare recessive conditions due to homozygous mutations in a child of mixed-race parents raised the possibility of uniparental isodisomy (UPiD). Single Nucleotide Polymorphism (SNP) microarray analysis confirmed two segments of homozygosity on chromosome 1 (1p36.2 to p32.2 and $1 \mathrm{p} 12$ to $1 \mathrm{q} 31.1)$ of maternal ancestry, encompassing both HSD3B2 and CLCKNB.

The patient is currently 6 years old and is managed with high doses of oral sodium $(16 \mathrm{mmol} / \mathrm{kg} / \mathrm{day})$ and potassium supplements $(6.5 \mathrm{mmol} / \mathrm{kg} / \mathrm{day})$, hydrocortisone $\left(8 \mathrm{mg} / \mathrm{m}^{2} /\right.$ day $)$, fludrocortisone $(60 \mathrm{mcg} /$ day $)$ and indomethacin $(9 \mathrm{mg}$ three times daily). Her height is $113 \mathrm{~cm}(-0.57$ SDS) and weight is $18.3 \mathrm{~kg}(-0.26 \mathrm{SDS})$. Her initial clinical course was complicated by recurrent admissions secondary to severe electrolyte imbalance. Developmentally, she has slight speech and motor delay which are improving. There have been no concerns with her cognition. Her height SDS continues to show improvement with the current treatment (Figure 1). 


\section{Discussion}

The combination of two rare, recessively inherited disorders in the same patient, with a background of non-consanguinity is extremely rare, which raised the suspicion of UPiD. Uniparental disomy (UPD) occurs when two copies of a given genomic region are derived from a single parent. Gamete complementation and trisomic zygote rescue are two of the mechanisms implicated [5]. UPD can occur as heterodisomy, when the segments coming from the single parent are different (meiosis stage I error) or isodisomy (UPiD), in which two identical segments from one parental homologue are present (meiosis stage II error) [5]. A recent study of the prevalence of UPD, using computational detection of DNA segments that were identical by descent and runs of homozygosity, estimated the incidence to be approx. 1 in 2000 births. This was nearly twice the frequency previously thought [6]. A consequence of UPiD is that it allows two copies of a recessive mutation to be transmitted from a heterozygous carrier parent to the child in whom the mutation will be present in the homozygous state [5]. Isodisomy is a very rare cause of CAH due to 21-hydroxylase deficiency [7] but has been also reported in single families with $\mathrm{CAH}$ due to deficiencies of 11B-hydroxylase and 3B-hydroxysteroid dehydrogenase [8]. The latter was due to a complete isodisomy of chromosome 1 unmasking a deleterious missense mutation [9].

The hallmark of Bartter syndrome is hypokalemic, hypochloraemic metabolic alkalosis associated with high plasma renin activity and high aldosterone, while $\mathrm{CAH}$ due to HSD3B2 enzyme deficiency typically presents with hyponatremia and hyperkalaemia associated with high plasma renin activity and low aldosterone. Our patient's initial investigations at presentation showed a sodium of $127 \mathrm{mmol} / \mathrm{l}$ and potassium of $6.8 \mathrm{mmol} / /$ yet, interestingly, she had alkalosis $\left(\mathrm{pH} 7.54, \mathrm{PCO}_{2} 4.7 \mathrm{kpa}\right.$, 
$\mathrm{PO}_{2} 5.2 \mathrm{kpa}$, bicarbonate $29.9 \mathrm{mmol} / \mathrm{l}$ ), indicating an uncoupling of acid base homeostasis from sodium and potassium homeostasis. Elevated FE of $\mathrm{Cl}(>0.5 \%)$ was consistent with chronic salt wasting [10]. FE of $\mathrm{Na}$ was normal but this is to be expected with salt wasting disorders in steady state, since persistent salt wasting is incompatible with life and thus other reabsorptive pathways compensate to some degree. The TAL is usually not an important site of tubular $\mathrm{H}^{+}$secretion, as this is predominantly mediated in the collecting duct. However, the situation may change when salt reabsorption in the TAL is impaired. Experiments studying the mechanism of furosemide-induced urinary acidification in murine medullary TAL have shown that this drug triggered a substantial drop in intracellular $\mathrm{Na}+$ concentration, increasing the driving force for the $\mathrm{Na}^{+} / \mathrm{H}^{+}$exchanger (NHE3), resulting in urinary acidification [11]. Thus, with blocked salt reabsorption through NKCC2 (Na-K-Cl cotransporter) in the TAL, NHE3 comes to the forefront and reabsorbs $\mathrm{Na}$ in exchange for protons, which could explain the metabolic alkalosis in our patient [12]. While in our patient Bartter syndrome is caused by impaired function of the chloride channel CLCNKB, this indirectly affects NKCC2 because of the increased intracellular chloride concentration [13]

The patient subsequently developed persistent hypokalaemia, initially thought to be due to over replacement of fludrocortisone. Yet, while weaning of the medication resulted in a significant increase in the plasma renin concentration there was no improvement in the plasma potassium concentration, suggestive of a possible tubulopathy. Fludrocortisone promotes sodium reabsorption by causing the secretion of hydrogen and potassium in the collecting ducts. The administration of fludrocortisone, in our patient, to supplement the lack of mineralocorticoid due to the 
co-existing $\mathrm{CAH}$, was probably contributing to the persistence of the hypokalemic alkalosis, although these abnormalities persisted even when fludrocortisone was withheld for up to 36 hours. The exact mechanism of the apparent excess mineralocorticoid activity thought to be driving the persistent hypokalemic alkalosis in our patient is not entirely clear and challenges our current understanding of mineralocorticoid physiology.

We have located two reports of co-presentation of $\mathrm{CAH}$ and Bartter syndrome. Both are based on biochemistry and not genotyping. Both feature increased renin and aldosterone, so the mineralocorticoid pathway appears not to be impaired, in contrast to our case. Yabe et al. [14] interpret their serum steroid data on a female patient presenting at $17 y$ with short stature, primary amenorrhoea and episodes of salt craving as consistent with 21-hydroxylase deficiency, but $3 \beta$-hydroxysteroid dehydrogenase deficiency appears more likely: the ratios of pairs of $3 \beta$-hydroxy-5ene steroids over 3-oxo-4-ene steroids DHEA/androstenedione, pregnenediol/pregnanediol and 17-hydroxypregnenolone/17-hydroxyprogesterone are $4.5,8.1 \& 9.0$ respectively. Dexamethasone suppressed the marker steroids but not aldosterone. Hypokalaemia persisted and fractional calcium reabsorption was diminished. Hussain et al. [15] describe a male neonate with vomiting and dehydration. There was marked hypokalaemia, hyponatraemia, hypocalcaemia and metabolic alkalosis. After discharge on indomethacin and potassium supplements, he returned with further vomiting and dehydration. Elevated 17-hydroxyprogesterone and ACTH identified 21-hydroxylase deficiency; he improved on hydrocortisone.

In the newborn period, definitive diagnosis of HSD3B2 deficiency by urine steroid profile is difficult because the most diagnostic marker, pregnenetriol, derived from 17-hydroxypregnenolone, is at a relatively low level at this time. A marked increase 
of DHEA metabolites relative to cortisol metabolites is the best pointer and this was present (Table 1). Prematurity can give rise to a similar pattern and the emergence of pregnenetriol is also delayed. The increased 17-hydroxyprogesterone $(>110 \mathrm{nmol} / \mathrm{L})$ is explained by peripheral conversion of 17 -hydroxypregnenolone by HSD3B1 isoenzyme. This can lead to an erroneous identification of 21-hydroxylase deficiency [16] but this disorder is readily ruled in or out by urine profiling. This distinction can be made using steroid metabolite ratios [16] and these are included in Table 1. The relevant metabolites for ratio calculation were not detectable in the 12 day sample; in the later samples, most of the cortisol metabolites will have been derived from the maintenance hydrocortisone being given, so this will have diminished the ratios calculated over cortisol metabolites. Each of the 17hydroxyprogesterone metabolites are elevated, but not to the degree seen in 21 hydroxylase deficiency, while pregnenetriol shows a much greater degree of increase, which is greatly enhanced after synacthen. Excretion of 11oxopregnanetriol, the major metabolite of 21-deoxycortisol, is relatively much lower than in 21-hydroxylase deficiency. 21-Deoxycortisol is generated by intra-adrenal 11B-hydroxylation of excess 17-hydroxyprogesterone, so is not found in excess in HSD3B2 deficiency because the 17-hydroxyprogesterone has a peripheral origin [16].

Diagnosis of HSD3B2 deficiency can also be made by measurement of 17hydroxypregnenolone following ACTH stimulation [16]. Assays for this steroid and also 21-deoxycortisol are not widely available, but serum panelling by LC-MS/MS can provide simultaneous analysis of these and all the other intermediates useful in distinction of forms of $\mathrm{CAH}[17]$. 
The linear growth in our patient was poor initially but has subsequently improved (Figure 1).There have been reports from the literature of associated growth hormone (GH) deficiency in children with Bartter syndrome $[18,19]$. Studies have shown low serum GH and IGF-1 concentrations in potassium-depleted rats and administration of $\mathrm{GH}$ does not stimulate longitudinal growth in them without the correction of the hypokalaemia $[20,21,22]$ In addition, abnormalities of the maturation of chondrocytes have been demonstrated in potassium-depleted rats, which did not improve with exogenous $\mathrm{GH}$ administration in a hypokalaemic state [23]. As the linear growth in our patient is improving, there are no current concerns about GH deficiency.

\section{Conclusion}

The identification of a homozygous rare mutation in an offspring of nonconsanguineous parents, especially of different ancestral background should raise the suspicion of uniparental isodisomy. Despite identifying the genetic cause, hypokalemic alkalosis, the biochemical fingerprint of hyperaldosteronism in a child with $\mathrm{CAH}$ challenges our current understanding of mineralocorticoid-mediated effects in the collecting duct.

\section{Acknowledgement:}

We would like to acknowledge Julia Oakley for her contributions to the manuscript.

\section{Statement of Ethics:}

A written and informed consent has been obtained from the parents for publication of this case report

\section{Disclosure Statement:}

The authors have no conflicts of interest to declare. 
Funding sources: None

Author contributions: DG prepared the manuscript and edited the subsequent revisions. DB, CD, JA, NT,GR, HM, SS and MA were involved in the clinical management of the patient and contributed to revisions of the manuscript at various stages. All authors have approved the final version of the manuscript. 


\section{References}

1. Miller $\mathrm{W}$ L, Auchus $\mathrm{R} \mathrm{J}$. The molecular biology, biochemistry and physiology of human steroidogenesis and its disorders. Endocr Rev. 2011 Feb;32(1):81-151.

2. Simard J, Moisan AM, Morel Y. Congenital adrenal hyperplasia due to 3betahydroxysteroid dehydrogenase/Delta(5)-Delta(4) isomerase deficiency. Semin Reprod Med. 2002 Aug; 20(3):255-76.

3. Kleta R, Bockenhauer D Salt-Losing Tubulopathies in Children: What's New, What's Controversial? J Am Soc Nephrol. 2018 Mar;29(3):727-39

4. Ashton EJ, Legrand A, Benoit V, Roncelin I, Venisse A, Zennaro MC et al. Simultaneous sequencing of 37 genes identified causative mutations in the majority of children with renal tubulopathies. Kidney Int. 2018 Apr;93(4):961-7.

5. Robinson WP. Mechanisms leading to uniparental disomy and their clinical consequences. Bioessays. 2000 May; 22(5):452-9.

6. Nakka P, Pattillo Smith S, O'Donnell-Luria AH, McManus KF; 23andMe Research Team, Mountain JL, Ramachandran S, Sathirapongsasuti JF. Characterization of Prevalence and Health Consequences of Uniparental Disomy in Four Million Individuals from the General Population. Am J Hum Genet. 2019 Nov 7;105(5):921932.

7. Pignatelli D, Carvalho BL, Palmeiro A, Barros A, Guerreiro SG, Maçut D The Complexities in genotyping of congenital adrenal hyperplasia: 21-hydroxylase deficiency. Front Endocrinol (Lausanne) 2019 Jul;10:432. doi: 10.3389/fendo.2019.00432. eCollection 2019.

8. Matsubara K, Kagami M, Fukami M Uniparental disomy as a cause of pediatric endocrine disorders. Clin Pediatr Endrocrinol 2018 Jul;27(3)113-21

9. Panzer K, Ekhaguare OA, Darbro B, Cook J, Schelochkov OA Uniparental isodisomy of chromosome 1 unmasking an autosomal recessive 3-beta hydroxysteroid dehydrogenase type II-related congenital adrenal hyperplasia. J Clin Res Pediatr Endocrinol 2017 Jan;9(1):70-3

10. Walsh, PR, Tse, Y, Ashton, E, lancu, D, Jenkins, L, Bienias, M, Kleta, R, Van't Hoff, W, Bockenhauer, D: Clinical and diagnostic features of Bartter and Gitelman syndromes. Clin Kidney J, 11: 302-309, 2018

11. de Bruijn PI, Larsen CK, Frische S, Himmerkus N, Praetorius HA, Bleich M, et al. Furosemide-induced urinary acidification is caused by pronounced $\mathrm{H}+$ secretion in the thick ascending limb. Am J Physiol Renal Physiol.2015 Jul ;309(2):F146-53.

12. Ponce-Coria J, San-Cristobal P, Kahle KT,Vazquez N, Pacheco-Alvarez D, de Los Heros et al. Regulation of NKCC2 by a chloride-sensing mechanism involving the WNK3 and SPAK kinases. Proc Natl Acad Sci U S A. 2008 Jun ;105(24):8458-63.

13. Jeandron DD, Sahakitrungruang T. A novel homozygous Q334X mutation in the HSD3B2 gene causing classic 3ß-hydroxysteroid dehydrogenase deficiency: an unexpected diagnosis after a positive newborn screen for 21-hydroxylase deficiency. Horm Res Paediatr. 2012 Feb; 77(5):334-8. 
14. Yabe R, Mizuno K, Ojima M, Ogawa S, Hashimoto S, Kunii N, Kishino K, Tani $\mathrm{M}$, Niimura $\mathrm{S}$, Watari $\mathrm{H}$, et al. A case of 21-hydroxylase deficiency and Bartter's syndrome associated with a balanced 6-9 translocation. Nihon Naibunpi Gakkai Zasshi. 1986 Aug;62(8):843-56.

15. Hussain $S$ Neonatal Bartter syndrome in association with congenital adrenal hyperplasia - a rare combination. J Pak Med Assoc 2016 May;66(5):612-4

16. Rumsby G. 3ß-hydroxysteroid dehydrogenase/isomerase deficiency. In Disorders of Steroidogenesis. Guide to steroid profiling and biochemical diagnosis. Editors: $G$ Rumsby and G M Woodward. Springer 2019

17. Caulfield MP, Lynn T, Gottschalk ME, Jones KL, Taylor NF, Malunowicz EM, Shackleton $\mathrm{CH}$, Reitz RE, Fisher DA. The diagnosis of congenital adrenal hyperplasia in the newborn by gas chromatography/mass spectrometry analysis of random urine specimens. J Clin Endocrinol Metab 2002 Aug; 87(8):3682-90.

18. Buyukcelik M, Keskin M, Kilic BD, Kor Y, Balat A. Bartter syndrome and growth hormone deficiency: three cases. Pediatr Nephrol. 2012 Nov; 27(11):2145-8.

19. Adachi M, Tajima T, Muroya K, Asakura Y. Classic Bartter syndrome complicated with profound growth hormone deficiency: a case report. J Med Case Rep. 2013 Dec;7:283.

20. Flyvbjerg A, Dorup I, Everts ME, Orskov H. Evidence that potassium deficiency induces growth retardation through reduced circulating levels of growth hormone and insulin -like growth factor I. Metabolism 1991 Aug; 40(8):769-75

21. Dorup I, Flyvbjerg A. Effects of IGF-I infusion on growth and muscle $\mathrm{Na}(+)-\mathrm{K}+$ pump concentration in K (+)-deficient rats. Am J Physiol. 1993 May;264:E810-E5

22. Hochberg Z, Amit T, Flyvbjerg A, Dorup I Growth hormone (GH) receptor and GHbinding protein deficiency in the growth failure of potassium-depleted rats. J Endocrinol.1995 Nov; 147(2):253-8

23. Gil-Peña H, Garcia-Lopez E, Alvarez-Garcia O, Loredo V, Carbajo-Perez E, Ordoñez FA, et al. Alterations of growth plate and abnormal insulin-like growth factor I metabolism in growth-retarded hypokalemic rats: effect of growth hormone treatment. Am J Physiol Renal Physiol 2009 Sep; 297(3):F639-F45 


\begin{tabular}{|c|c|c|c|c|c|c|c|}
\hline \multirow[t]{2}{*}{ Steroid (ug/L/mmol creatinine) } & \multirow[t]{2}{*}{12 days } & \multirow[t]{2}{*}{$\begin{array}{l}\text { Normal } \\
\text { mean }\end{array}$} & \multirow{2}{*}{$\begin{array}{l}\text { 1y } 2 \\
\text { months } \\
\text { baseline }\end{array}$} & \multirow{2}{*}{$\begin{array}{l}1 \text { y } 2 \\
\text { months } \\
6 \text { days } \\
\text { (Post } \\
\text { synacthen) }\end{array}$} & \multirow[b]{2}{*}{$\begin{array}{l}\text { Normal } \\
\text { Mean } \\
(n=15)\end{array}$} & \multicolumn{2}{|c|}{$\begin{array}{l}\text { Published [16] } \\
\text { Age 5-30 days }\end{array}$} \\
\hline & & & & & & $\begin{array}{l}\text { Cyp21 } \\
\text { Def } \\
\text { (range } \\
n=31 \text { ) }\end{array}$ & $\begin{array}{l}\text { HSD3B2 } \\
\text { Def } \\
(n=2)\end{array}$ \\
\hline DHEA metabolites & 2317 & 153 & 472 & 1647 & 14 & & \\
\hline Cortisol metabolites (FM) & 13 & 35 & 2110 & 1416 & 1831 & & \\
\hline 17-hydroxypregnanolone* & & & 87 & 175 & 11 & & \\
\hline Pregnanetriol $^{*}$ & & & 430 & 1715 & 27 & & \\
\hline 11-Oxopregnanetriol ${ }^{*}$ & & & 2 & 12 & 1 & & \\
\hline Pregnenetriol+ & & & 972 & 8614 & 12 & & \\
\hline Androstenedione metabolites & & & 79 & 183 & 25 & & \\
\hline \multicolumn{8}{|l|}{ Ratios } \\
\hline 17-hydroxypregnanolone x 100/FM & & & 4.1 & 12.4 & 0.6 & $248-1470$ & 75,224 \\
\hline Pregnanetriol x 100/FM & & & 20.4 & 121 & 1.5 & $77-1344$ & 6,16 \\
\hline 11-Oxopregnanetriol x 100/FM & & & 0.1 & 0.8 & 0.05 & $83-961$ & 5,8 \\
\hline Pregnenetriol x 100/FM & & & 46.1 & 608 & 0.65 & $<288$ & 149,356 \\
\hline Pregnenetriol/11-oxopregnanetriol & & & 486 & 718 & 12 & $<1.5$ & 32,43 \\
\hline DHEA metabolites/cortisol metabolites & 180 & 4 & 0.2 & 1.16 & 0.008 & & \\
\hline $\begin{array}{l}\text { DHEA metabolites/androstenedione } \\
\text { metabolites }\end{array}$ & & & 5.9 & 9.0 & 0.6 & & \\
\hline
\end{tabular}

Table 1. Urinary steroid excretion by the patient. The following metabolites were included: DHEA metabolites: (12 days) 16 $\alpha-$

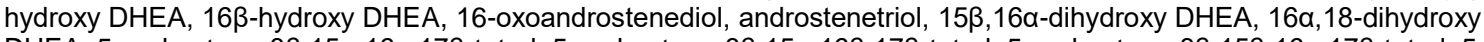

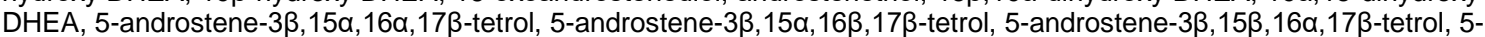

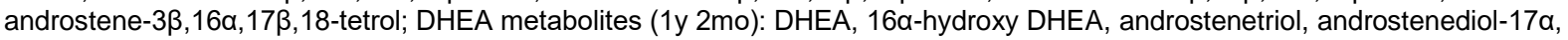
androstenediol-17 $\beta$; cortisol metabolites: (12 days) tetrahydrocortisol, $\alpha$-cortolone, $\beta$-cortolone, $1 \beta$-hydroxytetrahydrocortisol, $1 \beta$-hydroxy- $\beta$-cortolone, $6 \alpha$-hydroxytetrahydrocortisone, $6 \alpha$-hydroxy- $\alpha$-cortolone, $6 \alpha$-hydroxy- $\beta$-cortolone; cortisol metabolites (1y $2 \mathrm{mo}$ ): tetrahydrocortisone, $\alpha$-cortolone, $\beta$-cortolone, tetrahydrocortisol, allo-tetrahydrocortisol, $\alpha$-cortol, $\beta$-cortol; androstenedione metabolites: androsterone, aetiocholanolone. *:17-hydroxyprogesterone metabolites; +: 17hydroxypregnenolone metabolite. 
Figure Legend:

Figure 1: Growth chart showing improvements in height SDS with treatment

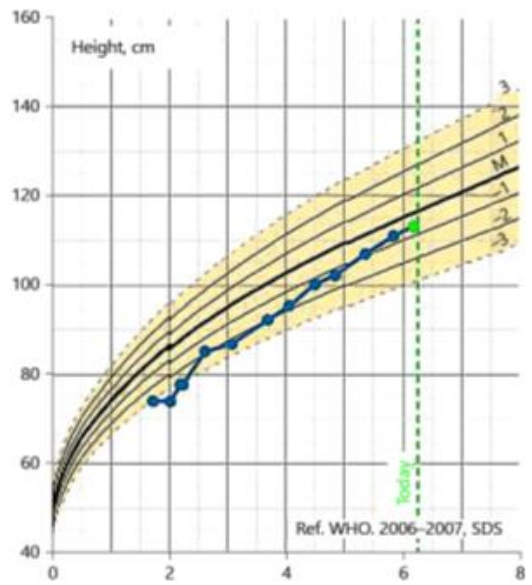

